\title{
Article
}

\section{Collective List-only Entity Linking: A Graph-based Approach}

\author{
${ }_{4}$ Weixin Zeng ${ }^{1}(\mathbb{D})$, Xiang Zhao ${ }^{1,2, *(1)}$ and Jiuyang Tang ${ }^{1,2}$ \\ 1 National University of Defense Technology, China \\ 2 Collaborative Innovation Center of Geospatial Technology, China \\ * Correspondence: xiangzhao@nudt.edu.cn; Tel.: +86-731-84576563
}

\begin{abstract}
List-only entity linking is the task of mapping ambiguous mentions in texts to target entities in a group of entity lists. Different from traditional entity linking task, which leverages rich semantic relatedness in knowledge bases to improve linking accuracy, list-only entity linking can merely take advantage of co-occurrences information in entity lists. State-of-the-art work utilizes co-occurrences information to enrich entity descriptions, which are further used to calculate local compatibility between mentions and entities to determine results. Nonetheless, entity coherence is also deemed to play an important part in entity linking, which is yet currently neglected. In this work, in addition to local compatibility, we take into account global coherence among entities. Specifically, we propose to harness co-occurrences in entity lists for mining both explicit and implicit entity relations. The relations are then integrated into an entity graph, on which Personalized PageRank is incorporated to compute entity coherence. The final results are derived by combining local mention-entity similarity and global entity coherence. The experimental studies validate the superiority of our method. Our proposal not only improves the performance of list-only entity linking, but also opens up the bridge between list-only entity linking and conventional entity linking solutions.
\end{abstract}

Keywords: list-only entity linking; named entity disambiguation; graph-based approach

\section{Introduction}

Entity Linking (EL) is the task of detecting corresponding named entities for ambiguous mentions in text. Mention refers to character string, such as Jackson in the example in Figure 1, the true meaning of which needs to be determined by being linked to an entity, such as the basketball coach Phil Jackson. Traditional EL methods leverage knowledge bases (KBs), which offer rich semantic information of entities, for robust and accurate disambiguation process. Nevertheless, despite the effectiveness of knowledge-based EL, it might not be applicable in situations where there is insufficient information of entities, such as entity lists.

Entity list, as is often the case, consists of a group of closely-related entities, and it exists in various information sources [1]. In contrast to KBs, where complete structure of entities facilitates almost all entity-related tasks, entity list minimizes necessary information to mere co-occurrences of interrelated entities, thus serving as a light-weight alternative in terms of describing entity correlations.

Entity lists can be found useful, for instance, in the scenario concerning detection of emerging stock names. When investors search new stock names in Wikipedia ${ }^{1}$, a frequently updated KB, chances are that there are no corresponding items. In fact, as is shown in [2], for a dataset including 2,468 stock names, merely 340 of them can be found in Wikipedia. Nevertheless, those stocks can be found co-occurring with others in stock lists on financial websites. Thus, the stock lists will be of great use if people have doubts concerning new stocks. There are much more similar situations, such as searching for specific car brand or collecting information about bars in a small town, where the knowledge about target entities are sparse.

1 https://www.wikipedia.org/ 


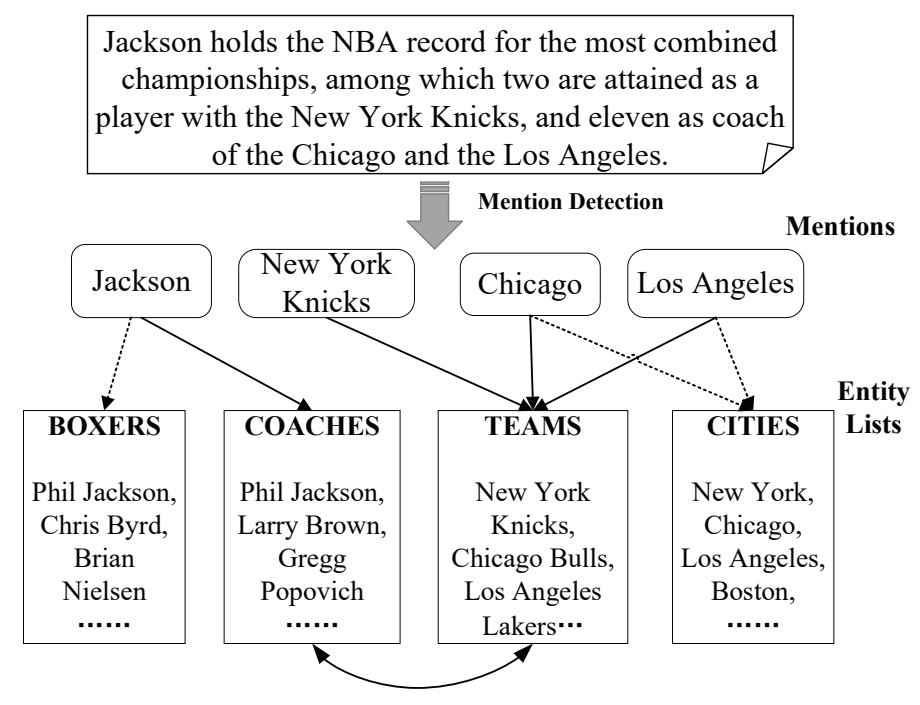

Figure 1. Example of list-only EL

Consequently, the demand for list-only EL emerges [1], which targets at solving the problem of mapping ambiguous mentions to entity lists (rather than KBs); Figure 1 describes an example of list-only EL problem. State-of-the-art method [1] addresses the challenge by merely considering the local compatibilities between mentions and entities to determine matching pairs, whereas neglecting the global coherence among entities.

Example 1. As shown in Figure 1, there is a piece of text with mentions Jackson, New York Nicks, Chicago and Los Angeles; and there are 4 sample entity lists to be linked to, namely Boxers, Coaches, Teams and Cities. The task of list-only EL is to link mentions to correct entities in the entity lists. It can be seen that entity Chicago and entity Los Angeles in entity list Cities have the same name strings with mention Chicago and mention Los Angeles in the text. Because of the high mention-entity compatibility, existing method tends to map mentions Chicago and Los Angeles to entities Chicago and Los Angeles in the entity list featured Cities. However, the true entities for them are Chicago Bulls and Los Angeles Lakers in entity list Teams. Furthermore, it is hard for current method to decide which entity that mention Jackson should be linked to, since there are two possible candidate entities with the same name Phil Jackson and they are in different entity lists.

Moreover, we observe from the dataset currently used for empirical study that each document only contains one mention for disambiguation, which may not reflect the reality well. A pragmatic scenario may look like the example in Figure 1, where there are four mentions to be disambiguated. Therefore, we contend that the existing evaluations could be inappropriate and need a redesign.

In short, the shortcomings of the existing list-only EL solution is two-fold:

- Entity coherence within or across entity lists were overlooked and not leveraged; and

- Results were supportless for lack of appropriate dataset and deliberate experiment design.

We close the gap and address the deficiencies in this article. In particular, we propose to solve list-only EL task by taking account of the correlations in entities and converting the disambiguation problem to a graph problem. We show the merits of graph-based list-only EL by referring to the example in Figure 1. It is easy to map mention New York Knicks to entity New York Knicks in the entity list featured Teams. Then by considering the interdependence of entities in the same list Teams, mention Chicago will be mapped to entity Chicago Bulls, and mention Los Angeles will be mapped to entity Los Angeles Lakers. Additionally, by further taking into account cross-dependence of entities across different entity lists, entity Phil Jackson in the Coaches entity list, rather than entity Phil Jackson in the Boxers entity list, will be chosen as the target entity for mention Jackson. 
To implement graph-based list-only EL, we mainly carry through the following three steps. (1) Pre-processing - including an optional named entity recognition process and the candidate entity generation process. This step formalizes raw texts and produces mentions and candidate entities as inputs for later steps. (2) Entity information enrichment. The descriptions of entities are enriched by collecting representative texts from the inputs, which in turn enable the establishment of coherence among entities. (3) Graph-based entity disambiguation. An entity graph is constructed by integrating outputs from earlier steps. We propose a graph-based algorithm Gloel, which implements Personalized PageRank to determine how likely an entity is the target entity by taking into consideration both coherences among entities, and compatibilities between mentions and entities. The output is a list of pairs comprising mentions and their most possible entities.

Furthermore, we put forward a new procedure to construct datasets applicable to evaluating list-only EL. The experimental results in this new dataset validate the effectiveness of graph-based linking, a popular method of collective linking, and the in-depth analysis shows that compared with existing list-only linking method, our graph-based solution achieves better performance in list-only EL task.

Contributions. The main contributions of this article can be summarized into three ingredients:

- We motivate to revise list-only EL by taking into account relations between entity lists, i.e., global coherence, in addition to local compatibilities between mentions and entities.

- We tackle the problem by a graph-based method and offer a new algorithm Gloel, where Personalized PageRank is adopted to capture global coherence among candidate entities.

- A new dataset construction procedure is presented to cater to the redefined task, and Gloel is experimentally evaluated on top of it, and shown to outperform state-of-the-art method.

Organization. This paper is organized as follows. In Section 2, the new definition of list-only EL problem and the methodology, which contains three steps, are elaborated. New dataset construction and experiment results are detailed in Section 3. Section 4 summarizes related work and bridges list-only EL with conventional KB-oriented EL, followed by conclusion in Section 5.

\section{Methodology}

We start with defining the proposed problem. Existing work defined list-only EL as mapping a single mention $m_{i}$ in document $d_{i}$ to the corresponding entity $e_{i, j} \in E_{j}$ in the entity lists. Nonetheless, on the one hand, in most real-life documents, there are more than one mention, differentiating this definition from reality. On the other hand, the ambiguity between entity lists is not stressed, which can turn the problem of mapping mentions to a group of highly ambiguous entities into determining whether the mentions have corresponding entities in the entity lists. And the latter also deviates from the original motivation of EL task, which centres on disambiguating mentions from several possible meanings. We will further elaborate the definition of ambiguity between entity lists via mathematical equations in Section 3.

As a consequence, it is vital to extend the definition of this task so as to cater to broader scenarios. Specifically, we formalize list-only EL problem as follows.

Definition 1 (List-only entity linking). Given a set of documents $D=\left\{d_{1}, \ldots d_{n}\right\}$, each of which contains a set of mentions $M_{i}=\left\{m_{i 1}, \ldots m_{i s}\right\}$, an ambiguous set of entity lists $\mathcal{E}=\left\{E_{1}, \ldots E_{l}\right\}$, the task is to determine the most possible entity $e_{i j, k} \in E_{k}$ for each mention $m_{i j}$, or return NIL if there is no corresponding entity.

Note that the set of entity lists has to be ambiguous. In other words, for the majority of entities, there ought to be at least one more ambiguous entity in the entity lists.

We take the example in Figure 1 to explain the definition. There are four mentions to be disambiguated in the document. By utilizing list-only EL, mentions New York Knicks, Chicago, Los Angeles should be mapped to entities New York Knicks, Chicago Bulls, Los Angeles Lakers in the entity 
list featured Teams respectively, instead of New York, Chicago, Los Angeles in the entity list featured Cities. And mention Jackson should be linked to entity Phil Jackson in the Coaches entity list, rather than entity Phil Jackson in the Boxers entity list.

The specific procedure for graph-based list-only entity linking includes three steps, namely, pre-processing, entity information enrichment and graph disambiguation. The former two steps generate inputs, based on which the entity graph is constructed and Gloel is performed to determine results.

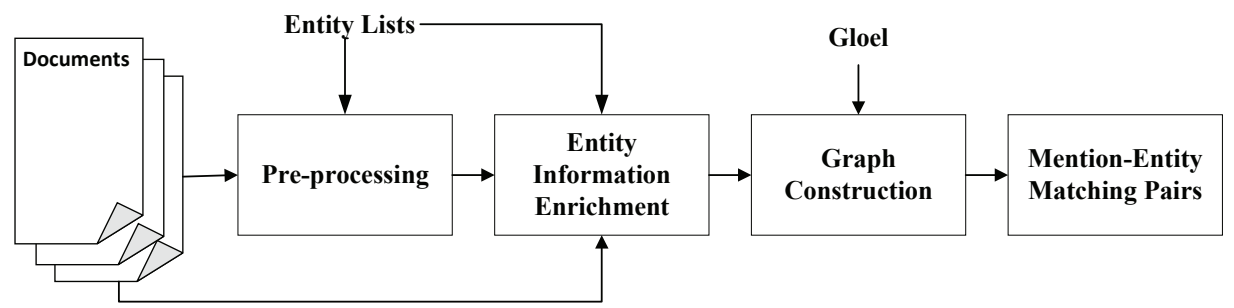

Figure 2. The flow diagram of graph-based list-only entity linking

\subsection{Preparation for Graph Input}

This subsection presents treatment of raw text data and generation of inputs for graph construction.

\subsubsection{Pre-processing}

In the pre-processing step, mentions in the text are detected and the candidate entities are also generated.

Specifically, the initial input for EL is a set of raw documents, either with specified mentions to be disambiguated or without. Under the circumstance where mentions are not pointed out, Named Entity Recognition (NER) should be harnessed to finish the mention detection task. State-of-the-art NER methods utilize Neutral Networks and Deep Learning techniques to achieve better performances, whereas they have not been widely used yet on account of the freshness and complexity. Instead, Stanford NER Tagger, a NER tool which is less accurate but maturer, embraces higher popularity in tasks involving but not focusing on NER. In our experiment, we have already extracted the mentions during dataset construction process.

After obtaining mentions, the following step is retrieving possible candidate entities for each mention. Take Figure 1 for instance, for mention Chicago, both entities Chicago Bulls and Chicago should be generated as candidates. In order to improve recall and generate more candidate entities, most KB-oriented EL methods tend to take advantage of name dictionaries embedded in KBs, or use alias dictionaries built from collecting Wikipedia redirecting and disambiguation pages. However, considering the limited number of target entities and sparse information of entity lists, we design a set of simple but efficient string matching rules for entity generation, as is shown in Table 1 . In the examples, the left are mentions while the right are candidate entities.

Table 1. String matching rules

\begin{tabular}{c|c}
\hline Rules & Examples \\
\hline \hline Containment & Chicago $\rightarrow$ Chicago Bulls \\
Partial Matching & President Trump $\rightarrow$ Donald Trump \\
Alternative & LA $\rightarrow$ Los Angeles \\
Names & National Capital $\rightarrow$ Washington, D.C. \\
\hline
\end{tabular}

The generated candidate entities for mention $m_{i j}$ are represented by $\operatorname{Can}\left(m_{i j}\right)$. Noteworthily, we adopt candidate-pruning policy to ensure that a mention will not have two or more candidates from the same list, since entity list is utilized to help candidate entity within it to compete with entities 
from other lists, and choosing among candidate entities from the same list will render coherence within entity list useless.

\subsubsection{Entity Information Enrichment}

Solely relying on co-occurrences between entities is not enough to establish relations among entities, let alone semantically bridge mentions with candidate entities. Therefore, we enrich information on the entity side by selecting representatives derived from input documents.

Given input documents $D=\left\{d_{1}, \ldots d_{n}\right\}$, the mentions $M_{i}=\left\{m_{i 1}, \ldots m_{i s}\right\}$ in each $d_{i}$, a set of entity lists $\mathcal{E}=\left\{E_{1}, \ldots E_{l}\right\}$, the enrichment process should collect a set of highly relevant and representative texts $T^{r}=\left\{t_{1}^{r}, \ldots t_{h}^{r}\right\}$ around mentions for $E_{r}$, which is achieved by harnessing co-occurrences of entities in the same entity list.

Specifically, the idea is that, since a document is not only composed of mentions, but also a lot of other irrelevant information, we merely extract the texts around all mentions in all documents as candidate representatives $\tau$ to avoid noisy information. If a candidate representative $t_{p} \in \tau$ contains many entity names from the same entity list $E_{r}$, chances are that it indeed shares the same category or topic with entity list $E_{r}$, and the mention $m_{p}$ in candidate representative $t_{p}$ is thus much more likely to refer to the candidate entity from $E_{r}$. Consequently, $t_{p}$ is a representative of $E_{r}$ and the text in $t_{p}$ can be used to enrich the textual descriptions of entities in $E_{r}$.

We further illustrate the method in Figure 3. Note that in each candidate representative, the bold text represents a mention, and the rest texts are its surroundings. Given an entity list Cities and the entity Chicago, the goal is to collect relevant representatives for Chicago from documents, which are then used to enrich representatives of entity list Cities. In Document: United Paramount Network, there are three candidate representatives, two of them contain name string Chicago. However, Candidate Representative 1 includes no extra name strings of other entities from the entity list, thus might not refer to Entity List Cities. In contrary, both New York and Los Angeles co-occur with Chicago in Candidate Representative 3, indicating the high possibility that it is a true representative for Entity List Cities. Switching to Document: Gotham City, both Candidate Representatives contain name string Chicago. Despite that Candidate Representative 4 is derived from mention Chicago, we cannot consider it as a representative due to lack of co-occurrences information. Conversely, containing several name strings from entity list Cities, Candidate Representative 5 is chosen as a representative, even though it is built surrounding mention Detroit. 


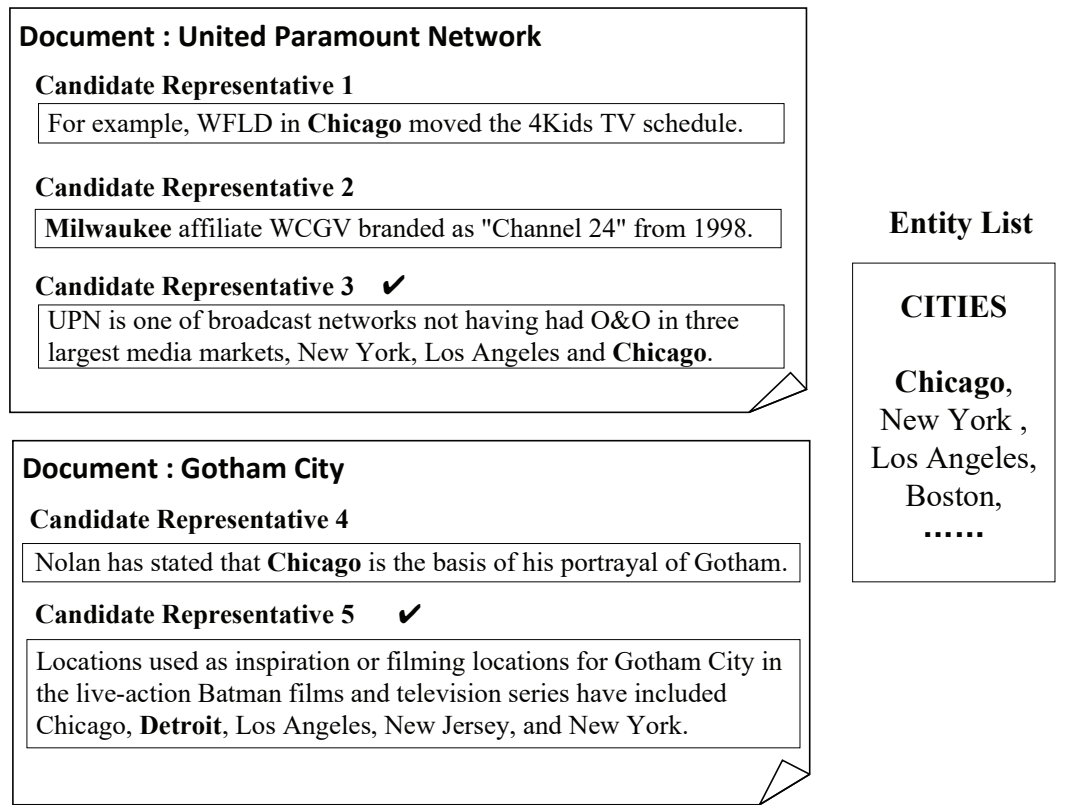

Figure 3. Example of entity information enrichment.

\subsection{Graph Construction and Disambiguation}

In this subsection, we illustrate the construction of candidate entity graph, followed by the description of our proposed algorithm Gloel, which takes advantage of Personalized PageRank so as to determine target entities.

\subsubsection{Graph Construction}

Through the pre-processing step, mentions and their candidate entities are obtained. Then after enriching textual descriptions in the entity side, the compatibility score between each mention and corresponding candidate entity can be calculated in terms of text similarity. Previous list-only EL ranks the candidate entities for each mention merely based on mention-entity compatibility scores, thereby producing the results accordingly. We argue that the judgement simply depending on compatibility score is not convincing enough because the coherence among entities is ignored, which plays an indispensable role in the linking process. For instance, as is shown in Figure 1, it is easy to map mentions Chicago and Los Angeles to the Cities entities Chicago and Los Angeles due to the short text information and high name string similarity. Provided that the candidate entity coherence is considered, the high interdependence among Teams entities New York Knicks, Chicago Bulls, Los Angeles Lakers will lead to the correct answers for mentions Chicago and Los Angeles.

To better capture the correlations among entities, similar to many existing KB-based EL methods, we construct an entity graph, which is depicted in Figure 4. The definition of entity graph is defined as follows: 


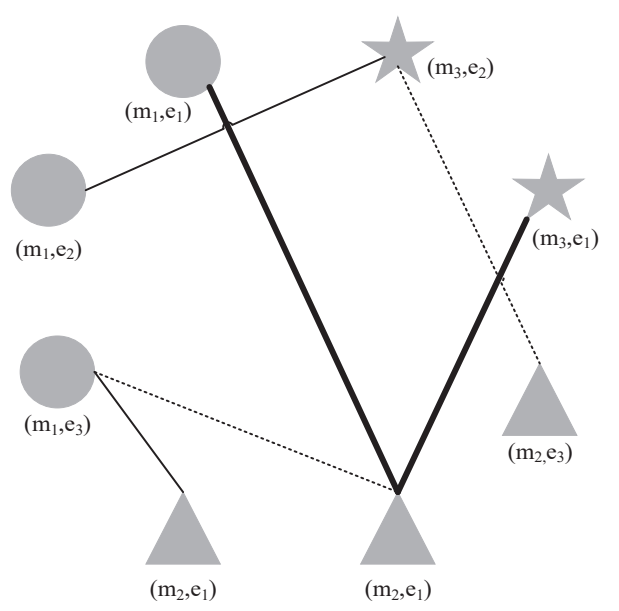

Figure 4. Entity graph.

Definition 2 (Entity graph). An entity graph $G=\{V, E\}$ is a weighed graph, in which the nodes $V$ represent all candidate entities, with their source mentions specified, and edges $E$ include relations between entities.

It is noteworthy that we differentiate the mentions with identical name strings even though they might appear in the same document, and similarly, by specifying the source mention of nodes, the candidate entities with the same name but generated from different mentions are also treated differently. In this way, the situations where there are duplicate nodes, either caused by mentions or entities, can be avoided. In the mathematical form, we represent the $r$-th candidate entity for mention $m_{i j}$ in document $d_{i}$ as $e_{i j, r}$, which clearly shows the source mention $m_{i j}$ of candidate entity $e_{i j, r}$.

With reference to edges, following the tradition in KB-based EL and adapting it to list-only problem, we connect two nodes with an edge under three circumstances: 1) The name strings of the two entities are in the same entity list $E \in \mathcal{E}$, and in this case, the edge weight is defined as $1 ; 2$ ) The name strings of the two entities simultaneously appear in at least one candidate representative $t \in \tau$ ; 3) The name strings of the other entities in the entity lists these two entities separately belong to, simultaneously appear in at least one candidate representative $t \in \tau$. The first two kinds of relations are termed as explicit relations, while the third method of adding edges among entities, named implicit relations mining, leverages the unique characteristic of entity list - that the rest entities $E_{i}^{\prime}=E_{i} \backslash\left\{e_{j}\right\}$ in the same entity list $E_{i}$ can help mine more correlations for entity $e_{j}$ even if $e_{j}$ is in the long tail. As for edge weight, which is defined below, takes into account both explicit and implicit relations between entities. Furthermore, the edges among candidate entities with the same source mention are pruned so as to eliminate the influence generated by competitors themselves.

We further assign initial node weight ini(v) and edge weight on the graph. The initial node weight $\operatorname{ini}(v)$ is defined as the compatibility score between candidate entity and its source mention, while edge weight is determined by relation score between the entities on the two sides of the edge. The specific approaches to calculate compatibility score and relation score are:

Compatibility score. Given a document $d_{i}$, and $m_{i j}$, a mention contained in $d_{i}$, suppose $e_{i j, r} \in E_{r}$ is a candidate entity for $m_{i j}$ and $T^{r}=\left\{t_{1}^{r}, \ldots t_{h}^{r}\right\}$ is the set of representative texts for $E_{r}$. The compatibility score $\phi\left(m_{i j}, e_{i j, r}\right)$ can be measured by the following equation

$$
i n i\left(m_{i j}, e_{i j, r}\right)=\phi\left(m_{i j}, e_{i j, r}\right)=\frac{1}{\left|T^{r}\right|} \sum_{p=1}^{\left|T^{r}\right|} \operatorname{Sim}\left(m_{i j}, t_{p}^{r}\right) .
$$

Since entities in the same entity list share the same representative texts, which are collected according to the method proposed in Section 2.1.2, calculating compatibility between a pair of mention 
$m_{i j}$ and candidate entity $e_{i j, r} \in E_{r}$ can be converted to computing the average text similarity Sim between texts surrounding mention $m_{i j}$ and all the text representatives $T^{r}$ of candidate entity $e_{i j, r}$.

There are many ways to measure text similarity Sim and in this paper, we choose to compute the similarity between embedding vectors of two texts, which is represented as $E\left(m_{i j}, t_{p}^{r}\right)$. Additionally, we also regard the name string similarity between mention and candidate entity as an appropriate indicator, and it is denoted as $N\left(m_{i j}, e_{i j}, r\right)$. Thus, the Compatibility score equation is converted to

$$
\phi\left(m_{i j}, e_{i j, r}\right)=\alpha N\left(m_{i j}, e_{i j}, r\right)+\beta \frac{1}{\left|T^{r}\right|} \sum_{p=1}^{\left|T^{r}\right|} E\left(m_{i j}, t_{p}^{r}\right) .
$$

In the equation above, $\alpha$ and $\beta$ are the weight coefficients balancing the importance of text similarity and name string similarity.

Relation score. Given two entities $e_{i}^{p} \in E_{p}, e_{j}^{q} \in E_{q}$ (We merely consider relationships among entities when calculating Relation Score, which is mention-irrelevant, thus we neglect the mention here), the Relation Score is denoted in the following equation

$$
\operatorname{Rel}\left(e_{i}^{p}, e_{j}^{q}\right)= \begin{cases}\eta O\left(e_{i}^{p}, e_{j}^{q}\right)+\frac{\theta}{M} \sum_{u}^{E_{p}-i} \sum_{v}^{E_{q}-j} O\left(e_{u}^{p}, e_{v}^{q}\right), & p \neq q \\ 1, & p=q,\end{cases}
$$

where $O\left(e_{i}, e_{j}\right)=\frac{\left|\operatorname{Occur}\left(e_{i}\right) \cap \operatorname{Occur}\left(e_{j}\right)\right|}{\left|\operatorname{Occur}\left(e_{i}\right) \cup \operatorname{Occur}\left(e_{j}\right)\right|}, \operatorname{Occur}(e)=\{t \mid e \in t, t \in \tau\}$, and $M=\left(\left|E_{p}\right|-1\right)\left(\left|E_{q}\right|-1\right)$.

We illustrate equations above as follows: $\operatorname{Occur}(e)$ denotes the occurrences of entity $e$ in All candidate representatives $\tau$, since compared with noisy textual information contained in the whole documents, merely considering texts around mentions (candidate representatives) can improve the accuracy. The Co-occurrence Frequency $O\left(e_{i}, e_{j}\right)$ of two entities $e_{i}$ and $e_{j}$ is defined as the number of candidate representatives they both occur in, divided by all the candidate representatives they either occur in together, or separately. As for the Relation $\operatorname{Score} \operatorname{Rel}\left(e_{i}^{p}, e_{j}^{q}\right)$ of two entities $e_{i}^{p} \in E_{p}, e_{j}^{q} \in E_{q}$, if $p$ equals $q$, which means $e_{i}^{p}$ and $e_{j}^{q}$ are from the same entity list, we set the relation score as 1 . Otherwise, the score is composed of two parts. The first component is the direct Co-occurrence Frequency of these two entities, multiplied by a weight factor $\eta$, which indicates explicit relations. While the implicit relations are represented by indirect Co-occurrence Frequency, the second component with a coefficient $\theta$, which takes into account the co-occurrences of the rest entities in $E_{p}$ and $E_{q}$ in a pair-wise fashion.

Furthermore, as is shown in Figure 4, there are three kinds of lines. The bold line represents that entities on the two sides are in the same entity list, and the Relation Score is 1. The dotted line denotes that two entities merely have implicit relations, while the normal line requires that there are explicit relations between entities.

It is noteworthy that, different from traditional KB-oriented EL problem which merely considers the direct relations between two entities, we extend the definition by taking into account the contribution made by relations between two entity lists as well, and represent them as implicit relations of two entities. The detailed approach to quantitatively describe the implicit relations is embodied in the equations above.

\subsubsection{Ranking Mention-entity Pairs}

Given a weighed entity graph $G_{i}$ of document $d_{i}$, the target is to find for each mention $m_{i j}$ in document $d_{i}$ the most likely entity $e_{i j, k}$ from a group of entities. In line with popular methods proposed in KB-oriented EL [3], we propose graph-based list-only entity linking algorithm, namely Gloel, which utilizes Personalized PageRank to depict the coherence among candidate entities.

Specifically, we assign a vector $p\left(v_{s}\right)$ with length $n$ to each node $v_{s}$ to represent the results of a PageRank process starting from $v_{s}$. To better capture the coherence among entities within the same document, instead of regarding the similarity between the vectors of nodes as the coherence score, we 
define it as how a candidate entity fits in the document. To enable the definition, a $n$-length vector $p\left(d_{i}\right)$ is also assigned to document $d_{i}$, representing the results of the PageRank process initiating from a group of unambiguous nodes. Consequently, the coherence score of a candidate entity $e_{i j, r}$ for mention $m_{i j}$ in document $d_{i}$ is defined as

$$
\psi\left(e_{i j, r}, d_{i}\right)=\frac{p\left(v_{i j, r}\right) p\left(d_{i}\right)}{\left|p\left(v_{i j, r}\right)\right|\left|p\left(d_{i}\right)\right|} .
$$

We first elaborate the random walk process initiating from a single node, then extend it to calculating document PageRank vector. The PageRank algorithm, based on random walk theory, is firstly proposed to measure the importance of web pages by counting the number and quality of links to this page. It has been applied to EL problems in recent years, and has achieved great performance [3-6]. The basic elements of PageRank include initial vector $r^{0}$, transition matrix $A$, and preference vector $s$. Note that in our method, $r^{0}=s$.

Transition Matrix $A$ is the same in both individual and collective processes, the value at $i$ th row and $j$ th column is defined as

$$
A_{i j}=\frac{\operatorname{Rel}\left(e_{i}, e_{j}\right)}{\sum_{e_{k} \in \operatorname{Edges}\left(e_{i}\right)} \operatorname{Rel}\left(e_{i}, e_{k}\right)} .
$$

where $\operatorname{Edges}\left(e_{i}\right)$ represents the edges connected to entity $e_{i}$.

When computing the vector $p\left(v_{t}\right)$ for a single node $v_{t}, r^{0}=s=(0 \ldots 0,1(t \text { th }), 0 \ldots)_{n}$, which means that $r^{0}$ and $s$ are identical $n$-length vectors, the position $t$ of the vector is assigned with 1 and the rest are endowed with 0 .

The situation is slightly more complicated as for document PageRank vector $p\left(d_{i}\right)$. Firstly, we regard a candidate entity $e_{i j, r}$ as a unambiguous one if it satisfies one of the following conditions:

1. $e_{i j, r}$ is the only candidate entity of mention $m_{i j}$ and $i n i\left(e_{i j, r}\right)$ is above threshold $\mu$. The unambiguous entities of this kind is endowed with initial weight $\lambda$.

2. When there are more than one candidate entities and $e_{i j, r}$ is the candidate entity with the largest initial value, suppose $e_{i j, r}^{\prime}$ is the candidate entity with the second largest initial value. It suffices that $\operatorname{ini}\left(e_{i j, r}\right)-i n i\left(e_{i j, r}^{\prime}\right) \geq \nu$. The initial weight of this kind is $\kappa$.

3. If there are no candidate entities meeting the conditions, all the candidate entities will be added to the unambiguous entities set, with the same weight endowments.

After obtaining unambiguous entities set, the actual weight can be assigned via normalization of initial weight values. Note that in graph, unambiguous entities are presented as equivalent nodes, and by placing the actual weight of unambiguous nodes in the corresponding positions of the $n$-length vector, we can attain $r^{0}$ and $s$ for document accordingly. Furthermore, we adopt an iterative disambiguation approach. In other words, after erasing ambiguity for each mention, the chosen result entity will be regarded as unambiguous and added to the unambiguous entities set, with initial weight of $\iota$. Afterwards, the document PageRank vector will be re-computed by utilizing the new unambiguous entities set.

With initial vector $r^{0}$, transition matrix $A$, and preference vector $s$ defined as above, the Personalized PageRank is presented as following

$$
r^{t+1}=(1-\rho) \times A \times r^{t}+\rho \times s
$$

In the equation above, $t$ represents the $t$ th iteration, and $\rho$ denotes the probability that the random walk process jumps out of the original iteration and starts from a new vector, which is usually set at 0.15. Normally, the restarting nodes are all nodes in the graph, and the weights in vector $s$ are the same, which equal to $\frac{1}{|V|}$. Nonetheless, in this work, vector $s$ is personalized and set as the same with initial vector, which means that the random walk merely restarts from the initial nodes, eliminating the effect from other nodes. When the iterative calculation reaches to a stage where $r^{k}$ does not change any more or the variation is within a minimal range, we consider that it converges and $p\left(v_{s}\right), p\left(d_{i}\right)$ are thereby attained. At last, we formalize the list-only EL problem in a mathematical way: 
Definition 3 (List-only entity linking in mathematical form). Given a set of documents D= $\left\{d_{1}, \ldots d_{n}\right\}$, each of which contains a set of mentions $M_{i}=\left\{m_{i 1}, \ldots m_{i s}\right\}$, an ambiguous set of entity lists $\mathcal{E}=\left\{E_{1}, \ldots E_{l}\right\}$, the task is to determine the most possible entity $e_{i j, k} \in E_{k}$ for each mention $m_{i j}$, and

$$
e_{i j, k}=\underset{e_{i j, r} \in \operatorname{Can}\left(m_{i j}\right)}{\arg \max }\left(\gamma \phi\left(m_{i j}, e_{i j, r}\right)+\delta \psi\left(e_{i j, r}, d_{i}\right)\right)
$$

where $\gamma$ and $\delta$ are two weight coefficients balancing the weight between mention-entity compatibility score and entity coherence score. NIL will be returned if there is no corresponding entity.

\section{Experiments and Results}

Considering the deficiency in current list-only EL dataset, we propose a similar but more comprehensive approach for dataset construction. Then our method is validated via experiments on this dataset and the merits are highlighted through comparison with state-of-the-art method.

\subsection{Dataset}

There are two shortcomings in current dataset [1]. For one thing, each document merely contains a single mention to be disambiguated, which does not fit in most real-life occasions. For another, the target entity lists are not ambiguous enough, giving rise to the situation that most mentions merely have one candidate entity, and the disambiguation problem is converted to judging whether this sole candidate entity is true or not. Take entity Apple in Company entity list shown in the dataset of [1], there is no other similar entities in the set of entity lists. As a result, when given a mention Apple, the candidate entity for it will only be Apple in Company entity list, and the problem is transformed into deciding whether the mention can be mapped to entity lists or not.

In order to overcome the deficiencies, we propose to mine target entity lists and collect documents. The entity lists can be constructed both manually and automatically, but the ambiguity must be ensured. Given two entity lists $E_{m}=\left\{e_{1, m}, \ldots e_{i, m}\right\}$ and $E_{n}=\left\{e_{1, n}, \ldots e_{j, n}\right\}$, for $E_{m}$, the ambiguity caused by the existence of $E_{n}$ is defined as

$$
\operatorname{Amb}\left(E_{m}, E_{n}\right)=\frac{1}{\left|E_{m}\right|} \sum_{e_{i, m} \in E_{m}} \underset{e_{j, n} \in E_{n}}{\operatorname{argmax}} a m b\left(e_{i, m}, e_{j, n}\right) .
$$

Note that $a m b\left(e_{i, m}, e_{j, n}\right)$ represents the ambiguity between two entities in different entity lists. Many approaches can be utilized to measure it, and in this paper, we harness the matching rules defined in the candidate entities retrieval section. If matching rules are satisfied, we endow 1 to $\operatorname{amb}\left(e_{i, m}, e_{j, n}\right)$. Otherwise, the value is determined by name string similarity. Furthermore, the reason why only the highest ambiguity value for $e_{i, m}$ is chosen lies in the fact that we merely need to assure $e_{i, m}$ has one ambiguous competitor to avoid the situation as the example above.

For the whole entity lists set $\mathcal{E}=\left\{E_{1}, \ldots E_{l}\right\}$, the ambiguity is denoted as

$$
A(\mathcal{E})=\frac{1}{|\mathcal{E}|} \sum_{E_{p} \in \mathcal{E}} \underset{E_{q} \in \mathcal{E} \backslash E_{p}}{\operatorname{argmax}} \operatorname{Amb}\left(E_{p}, E_{q}\right) .
$$

Again, for each entity list $E_{p}$, we only consider the highest ambiguity it has with the rest entity lists in $\mathcal{E}$, since constructing a entity lists set with high ambiguity between each pair of entity lists is nearly impossible. 
Referring to [1], we generated raw entity lists by utilizing NeedleSeek ${ }^{2}$, which were then filtered and processed according to the definition of ambiguity. At last, seven entity lists with 70 entities in total were generated. The ambiguity of newly-constructed entity lists set is 0.965 , calculated according to the equations given above, while the value ${ }^{3}$ for entity lists set in [1] is 0.267 . Part of the newly constructed entity lists are presented in Table 2.

Table 2. Part of entity lists.

\begin{tabular}{c|c}
\hline$E_{i}$ & Entities \\
\hline \hline 1 & Atlanta, Chicago, Boston, Houston, New York, Detroit, Cleveland, Atlanta, Milwaukee \\
2 & Atlanta Hawks, Chicago Bulls, Boston Celtics, Houston Rockets, Detroit Pistons... \\
3 & Atlanta Braves Chicago Cubs, Boston Red Sox, Houston Astros, Detroit Tigers... \\
4 & Toyota Camry, Ford Ikon, Tata Indica, Honda Accord, Hyundai Accent... \\
5 & Toyota, Ford, Tata Motors, Honda, Hyundai, Chevrolet, Porsche, Volkswagen, Buick... \\
6 & Cambridge, Oxford, St Andrews, Warwick, London, Edinburgh, Glasgow, Manchester... \\
7 & University of Cambridge, University of Oxford, University of St Andrews... \\
\hline
\end{tabular}

As for building the documents dataset, we emphasize that there have to be at least two mentions in the same document to enable the construction of candidate entity graph. Otherwise there will be no difference between independent linking method and the proposed collective linking method based on graph.

To be specific, we utilized wikilinks in Wikipedia to obtain the documents. For each entity $e_{i, k}$ in entity list $E_{k}$, its referent Wikipedia page was determined in the first place. For instance, the Wikipedia page of entity Atlanta is en.wikipedia.org/wiki/Atlanta. Then we randomly retrieved 1,000 Wikipedia pages directing at $e_{i, k}$ via the WhatLinksHere page. As for Atlanta, the url of its WhatLinksHere page is en.wikipedia.org/wiki/Special:WhatLinksHere/Atlanta. After conducting the same operation for all the entities in entity list $E_{k}$, the links appearing in at least three entities' 1,000 Wikipedia pages were selected and the web pages texts they refer to were considered as documents. In this way, we can affirm that each document involves at least three mentions. Table 3 describes the specific information of documents and mentions.

\subsection{Results and Analyses}

We compare Gloel and the method utilized in [1] (denoted as Independent) on the dataset we create. The results are shown in Table 4 and the settings of parameters are listed as follows: $\alpha=0.4, \beta=0.6, \eta=0.7, \theta=0.3, \gamma=0.5, \delta=0.5, \lambda=0.5, \kappa=0.4, \iota=0.3$.

The measurements we adopt are the same with the metrics in [7], namely Precision, Recall and F1. Precision takes into account all entity mentions that are linked by the system and determines the correctness. Recall on the other hand, considers all the mentions should be linked, and reflects the fraction of correctly linked mentions. F1 is a balanced indicator of Precision and Recall.

2 http://needleseek.msra.cn

3 Since the author did not offer the full entity lists information, we compute the ambiguity of the segmental entity lists presented in the previous work. 
Table 3. Dataset statistics

\begin{tabular}{c|cc}
\hline Target $E_{i}$ & \#documents & \#mentions \\
\hline \hline 1 & 156 & 731 \\
2 & 535 & 3,450 \\
3 & 528 & 3,054 \\
4 & 41 & 108 \\
5 & 151 & 742 \\
6 & 97 & 472 \\
7 & 115 & 564 \\
Total & 1,623 & 9,121 \\
\hline
\end{tabular}

Table 4. Experimental results on original dataset

\begin{tabular}{c|c|ccccccc|c}
\hline Method & & $E_{1}$ & $E_{2}$ & $E_{3}$ & $E_{4}$ & $E_{5}$ & $E_{6}$ & $E_{7}$ & Overall \\
\hline \hline \multirow{3}{*}{ Independent } & $\mathrm{P}$ & 0.914 & 0.996 & 0.998 & 0.818 & 0.998 & 0.667 & 0.997 & 0.962 \\
& $\mathrm{R}$ & 0.990 & 0.984 & 0.988 & 0.998 & 0.959 & 0.989 & 0.585 & 0.959 \\
& $\mathrm{~F} 1$ & 0.950 & 0.990 & 0.993 & 0.900 & 0.979 & 0.797 & 0.734 & 0.961 \\
& $\mathrm{P}$ & 0.997 & 1.000 & 0.998 & 0.931 & 1.000 & 0.675 & 0.997 & 0.974 \\
& $\mathrm{R}$ & 0.997 & 0.998 & 0.997 & 1.000 & 0.981 & 0.992 & 0.598 & 0.971 \\
& $\mathrm{~F} 1$ & $\mathbf{0 . 9 9 7}$ & $\mathbf{0 . 9 9 9}$ & $\mathbf{0 . 9 9 8}$ & $\mathbf{0 . 9 6 4}$ & $\mathbf{0 . 9 9 0}$ & $\mathbf{0 . 8 0 3}$ & $\mathbf{0 . 7 4 8}$ & $\mathbf{0 . 9 7 2}$ \\
\hline
\end{tabular}

Table 5. Experimental results on $50 \%$ corrupted dataset

\begin{tabular}{c|c|ccccccc|c}
\hline Method & & $E_{1}$ & $E_{2}$ & $E_{3}$ & $E_{4}$ & $E_{5}$ & $E_{6}$ & $E_{7}$ & Overall \\
\hline \hline \multirow{3}{*}{ Independent } & $\mathrm{P}$ & 0.968 & 0.735 & 0.987 & 0.167 & 0.968 & 0.867 & 0.639 & 0.766 \\
& $\mathrm{R}$ & 0.856 & 0.994 & 0.624 & 0.944 & 0.287 & 0.318 & 0.961 & 0.764 \\
& $\mathrm{~F} 1$ & 0.909 & 0.845 & 0.765 & 0.284 & 0.443 & 0.465 & 0.768 & 0.765 \\
& $\mathrm{P}$ & 0.641 & 1.000 & 0.998 & 0.943 & 0.967 & 0.555 & 0.995 & 0.910 \\
& $\mathrm{R}$ & 0.997 & 0.966 & 0.899 & 0.769 & 0.985 & 0.992 & 0.332 & 0.907 \\
& $\mathrm{~F} 1$ & 0.781 & $\mathbf{0 . 9 8 2}$ & $\mathbf{0 . 9 4 6}$ & $\mathbf{0 . 8 4 7}$ & $\mathbf{0 . 9 7 6}$ & $\mathbf{0 . 7 1 2}$ & 0.497 & $\mathbf{0 . 9 0 9}$ \\
\hline
\end{tabular}

We first report the results on original dataset. As is depicted in Table 4, Gloel outperforms independent EL method in all occasions, with a overall F1 gain at 1.1\%. Nevertheless, it is evident that both methods achieve high Precision, Recall and F1 scores. This can be justified that most mentions in the documents appear in the same name string form as the entity name strings. For instance, in documents containing mention referring to entity University of Cambridge, the name form of the mention is also University of Cambridge, thus the high name string similarity basically guarantees the correct matching and rules out the possibility of other candidate entities. Plus, this does not fit in situations of most text sources other than Wikipedia. In news reports concerning University of Cambridge, it constantly goes by the name Cambridge as in sentence Cambridge beats Oxford in terms of computer science. In these cases, the probability of generating result entity Cambridge is enhanced significantly and the disambiguation difficulty also rises up.

As a consequence, we corrupted the dataset to observe the corresponding results produced by these two methods. To achieve corruption and increase ambiguity, we replaced mention names of the entities in lists 2,3,5,7 to the corresponding ambiguous names in lists 1,4,6. For instance, the mention names Atlanta Hawks and Atlanta Braves were substituted by Atlanta. Considering the fact that after corruption, the name string similarity (in [1] the NER results) might be of no use and possibly lead to negative contributions, which was unfair for Independent results, we merely took into account the embedding vectors similarity in terms of mention-entity similarity calculation and altered the corresponding parameter setting.

The results of $50 \%$ corruption in Table 5 are generated after half of the entities' corresponding mention names in lists $2,3,5,7$ get replaced. For fair comparison, the parameters are optimized for 
separate methods. As can be seen, the gap between the results of Independent and Gloel widens. Gloel achieves better outcomes with overall F1 score at $90.9 \%$, while the overall $\mathrm{F} 1$ value of previous method is $76.5 \%$, hence validating the superiority of the proposed method.

We further conducted $25 \%$ and $75 \%$ corruption on the dataset and Figure 5 dynamically depicts the F1 scores of these two methods under corruption. With input texts getting more difficult, the results of EL based solely on mention-entity compatibility decline rapidly, while Gloel, a method based on graph, still yields robust results with smaller decreases.

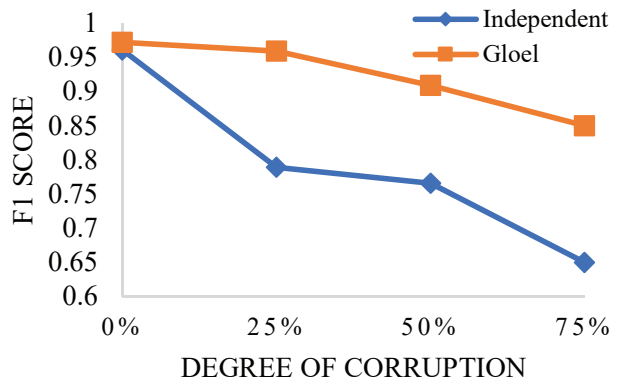

Figure 5. F1 score of independent and Gloel over corrupted dataset

\section{Related Work}

In this section, we brief related work, and discuss the differences and connections between list-only EL and traditional EL.

\subsection{List-only Entity Linking}

Over recent years, in accordance with the emergence of various text sources, EL tasks in new forms have been put forward. List-only EL task, first formally defined by Lin et al. [1], is the task of mapping mentions to a group of entity lists, rather than complete KBs. Lin et al. selected seed mentions for each entity list to bridge the gap between mentions and non-informative target entities, and then conducted the independent linking process to determine final results. Noticing that they merely harnessed entity lists co-occurrences information for generating entity descriptions, in this work, we further utilize the co-occurrences information to model entity relatedness and integrate it in the entity graph, which yields a more robust and accurate EL framework when confronting difficult input texts.

There are other new forms of EL problems which are similar to the list-only task. One is the Target Entity Disambiguation problem $[2,8]$. The main disparity is that the focus of Target Entity Disambiguation task lies in finding documents related to the entities given a entity list, whereas the starting point of list-only EL task is to eliminate the ambiguity in documents by using entity lists. Another similar task is the Named Entity Disambiguation with Linkless KBs [9]. Different from the mere entity lists in our task, there are still textual descriptions for entities in Linkless KBs.

\subsection{Knowledge Base Oriented Entity Linking}

Earlier works on EL focus on the situation where abundant information exists on the entity side. Specifically, KBs such as YAGO, Freebase and Wikipedia, offer rich semantic structures among entities as well as detailed textual descriptions, thus resulting in robust and accurate linking procedure. KB-oriented EL work can generally be divided into independent and collective methods.

In the former approach, mentions are disambiguated merely according the similarity between mentions and entities, and the problem is transformed into candidate entities ranking so as to obtain the most possible result. The similarity is mainly measured by lexical features such as bag-of-words of surrounding texts and statistical features such as prior popularities of entities. Then as for ranking process, unsupervised methods [10] calculate cosine similarities of feature vectors and output the results, whereas supervised approaches $[11,12]$ construct classifiers by training on annotated dataset, and the 
linking process is in the charge of classifiers when inputs are given. Although methods of this kind can achieve good results, semantic coherences within entities are neglected, which prove to be essential in improving overall performances.

With respect to collective linking methods in conventional EL task, most of them assume mentions in the same document are semantically coherent, which also should fit in the textual topic of the whole document. Therefore, the resulting entities also are expected to have high relatedness and the problem is in turn converted to find matching pairs maximizing the coherence. Cucerzan [13] proposed to harness Wikipedia categories to model coherence among entities, while Milne and Witten [14] reckoned normalized Google Distance as another useful tool for measurement, which was utilized by Kulkarni et al. [15] to form integer linear programming problem so as to collectively obtain results. Hoffart et al. [16] defined keyphrase relatedness to capture entity coherence, and proposed to construct a mention-entity graph, on which dense sub-graph generation algorithm was put forward to determine the sub-graph containing one-to-one mention-entity matches. The method of re-formalizing the linking problem by constructing mention-entity or entity-only graph distinguished itself among other works due to its capability to integrate both local similarity information between mentions and entities, along with the coherence information among entities. Based on this, several works [3-6] proposed and applied modified graph algorithm on the graph, which improved the disambiguation accuracy and the adaptability to difficult texts. Overall, the collective linking methods generally perform better than the independent counterparts in terms of conventional KB-oriented EL.

\subsection{Discussion on Differences and Connections}

There are indeed many similarities between these two lines of works, despite of the evident differences. The disparity mainly lies in the information on the entity side. Regarding conventional KB oriented EL, entities have rich and well-structured descriptions offered by KBs, in terms of both text description and internal links among entities[14-16]. Thereafter, researchers merely need to filter valuable information to improve linking results. In stark contrast, with respect to list-only scenarios, the mere information existing on the entity side is the co-occurrences among entity name strings in the same entity list, which in turn requires information mining and enrichment. In this paper, to avoid help from structured or semi-structured knowledge source, the dataset itself is leveraged to harvest the relevant relations among entities, thus fulfilling the entity information enrichment task.

Nevertheless, aside from information mining process, the methods utilized in conventional researches can be applied to this newly-defined problem and will achieve promising results. For instance, with disambiguation problem taking the form of graph, [3-6] can all be implemented.

Above all, the techniques developed in traditional EL also apply in list-only EL problem, and the extra work for the latter is to mine information on the entity side.

\section{Conclusion}

List-only entity linking task, as a new form of traditional EL problem, distinguishes itself by the sparse information on the entity side. In this work, on the one hand, we propose to utilize entity co-occurrences information to mine both textual description of entities and relations among entities, so as to enrich entity information. On the other hand, inspired by conventional EL methods, we construct an entity graph to capture relations among entities, on which the newly proposed algorithm Gloel is applied to obtain results. Similar to the situation in traditional EL, our approach, a collective EL method based on graph, outperforms independent EL on the dataset we create for fair comparison.

For future works, we plan to investigate two aspects. One is to consider the situation where an entity appears in more than one entity list. For instance, Washington, D.C. can appear in entity lists featured American Cities and Country Capitals. Another is to leverage word embedding techniques and deep neural networks to better model mention-entity compatibility and entity coherence.

Acknowledgments: This work was in part supported by NSFC under grants No. 61402494, 71690233 and 71331008, and NSF of Hunan Province under grant No. 2015JJ4009. 
Author Contributions: W. Zeng and X. Zhao conceived the problem and the method, conducted the experiments and wrote the paper; J. Tang managed the project and revised the paper.

Conflicts of Interest: The authors declare no conflict of interest. The founding sponsors had no role in the design of the study; in the collection, analyses, or interpretation of data; in the writing of the manuscript, and in the decision to publish the results.

\section{References}

1. Lin, Y.; Lin, C.; Ji, H. List-only Entity Linking. Proceedings of the 55th Annual Meeting of the Association for Computational Linguistics, ACL 2017, Vancouver, Canada, July 30 - August 4, Volume 2: Short Papers, 2017, pp. 536-541.

2. Cao, Y.; Li, J.; Guo, X.; Bai, S.; Ji, H.; Tang, J. Name List Only? Target Entity Disambiguation in Short Texts. Proceedings of the 2015 Conference on Empirical Methods in Natural Language Processing, EMNLP 2015, Lisbon, Portugal, September 17-21, 2015, 2015, pp. 654-664.

3. Guo, Z.; Barbosa, D. Robust Entity Linking via Random Walks. Proceedings of the 23rd ACM International Conference on Conference on Information and Knowledge Management, CIKM 2014, Shanghai, China,November 3-7, 2014, 2014, pp. 499-508.

4. Han, X.; Sun, L.; Zhao, J. Collective entity linking in web text: a graph-based method. Proceeding of the 34th International ACM SIGIR Conference on Research and Development in Information Retrieval, SIGIR 2011, Beijing, China, July 25-29, 2011, 2011, pp. 765-774.

5. Alhelbawy, A.; Gaizauskas, R.J. Graph Ranking for Collective Named Entity Disambiguation. Proceedings of the 52nd Annual Meeting of the Association for Computational Linguistics, ACL 2014, June 22-27, 2014, Baltimore, MD, USA, Volume 2: Short Papers, 2014, pp. 75-80.

6. Pershina, M.; He, Y.; Grishman, R. Personalized Page Rank for Named Entity Disambiguation. NAACL HLT 2015, The 2015 Conference of the North American Chapter of the Association for Computational Linguistics: Human Language Technologies, Denver, Colorado, USA, May 31 - June 5, 2015, 2015, pp. 238-243.

7. Shen, W.; Wang, J.; Han, J. Entity Linking with a Knowledge Base: Issues, Techniques, and Solutions. IEEE Trans. Knowl. Data Eng. 2015, 27, 443-460.

8. Wang, C.; Chakrabarti, K.; Cheng, T.; Chaudhuri, S. Targeted disambiguation of ad-hoc, homogeneous sets of named entities. Proceedings of the 21st World Wide Web Conference 2012, WWW 2012, Lyon, France, April 16-20, 2012, 2012, pp. 719-728.

9. Li, Y.; Tan, S.; Sun, H.; Han, J.; Roth, D.; Yan, X. Entity Disambiguation with Linkless Knowledge Bases. Proceedings of the 25th International Conference on World Wide Web, WWW 2016, Montreal, Canada, April 11 - 15, 2016, 2016, pp. 1261-1270.

10. Bunescu, R.C.; Pasca, M. Using Encyclopedic Knowledge for Named entity Disambiguation. EACL 2006, 11st Conference of the European Chapter of the Association for Computational Linguistics, Proceedings of the Conference, April 3-7, 2006, Trento, Italy, 2006.

11. Dredze, M.; McNamee, P.; Rao, D.; Gerber, A.; Finin, T. Entity Disambiguation for Knowledge Base Population. COLING 2010, 23rd International Conference on Computational Linguistics, Proceedings of the Conference, 23-27 August 2010, Beijing, China, 2010, pp. 277-285.

12. Mihalcea, R.; Csomai, A. Wikify!: linking documents to encyclopedic knowledge. Proceedings of the Sixteenth ACM Conference on Information and Knowledge Management, CIKM 2007, Lisbon, Portugal, November 6-10, 2007, 2007, pp. 233-242.

13. Cucerzan, S. Large-Scale Named Entity Disambiguation Based on Wikipedia Data. EMNLP-CoNLL 2007, Proceedings of the 2007 Joint Conference on Empirical Methods in Natural Language Processing and Computational Natural Language Learning, June 28-30, 2007, Prague, Czech Republic, 2007, pp. 708-716.

14. Milne, D.N.; Witten, I.H. Learning to link with wikipedia. Proceedings of the 17th ACM Conference on Information and Knowledge Management, CIKM 2008, Napa Valley, California, USA, October 26-30, 2008, 2008, pp. 509-518. 
15. Kulkarni, S.; Singh, A.; Ramakrishnan, G.; Chakrabarti, S. Collective annotation of Wikipedia entities in web text. Proceedings of the 15th ACM SIGKDD International Conference on Knowledge Discovery and Data Mining, Paris, France, June 28 - July 1, 2009, 2009, pp. 457-466.

16. Hoffart, J.; Yosef, M.A.; Bordino, I.; Fürstenau, H.; Pinkal, M.; Spaniol, M.; Taneva, B.; Thater, S.; Weikum, G. Robust Disambiguation of Named Entities in Text. Proceedings of the 2011 Conference on Empirical Methods in Natural Language Processing, EMNLP 2011, 27-31 July 2011, John McIntyre Conference Centre, Edinburgh, UK, A meeting of SIGDAT, a Special Interest Group of the ACL, 2011, pp. $782-792$. 